\title{
Elevated Serum Levels of Progranulin and Soluble Vascular Cell Adhesion Molecule-I in Patients with COVID-19
}

\author{
Shifei Yao ${ }^{1,2}$ \\ Nanning Luo 1,2 \\ Jiaoyang Liu',2 \\ He Zha' \\ Yuanhang $A i^{1,2}$ \\ Juan Luo ${ }^{1,2}$ \\ Shi Shi ${ }^{3}$ \\ Kaifeng Wu (D) ${ }^{1,2}$
}

'Department of Laboratory Medicine, Zunyi Medical University Third Affiliated Hospital/The First People's Hospital of

Zunyi, Zunyi, 563000, Guizhou, People's Republic of China; ${ }^{2}$ Scientific Research

Center, Zunyi Medical University Third Affiliated Hospital/The First People's Hospital of Zunyi, Zunyi, 563000, Guizhou, People's Republic of China; ${ }^{3}$ Department of Laboratory Medicine, The Fourth People's Hospital of Zunyi, Zunyi, 563000, Guizhou, People's

Republic of China
Correspondence: Kaifeng Wu Department of Laboratory Medicine/ Scientific Research Center, Zunyi Medical University Third Affiliated Hospital/The First People's Hospital of Zunyi, Zunyi, 563000, Guizhou, People's Republic of China

Tel +86 85I23II 6548

Email kiphoonwu@I26.com
Background: Severe acute respiratory syndrome coronavirus 2 (SARS-CoV-2) infection is associated with the angiocentric inflammation and angiogenesis, yet the molecules involved in this process remain to be determined.

Methods: We did a cross-sectional study of a cohort of patients with COVID-19 in Zunyi, China between February 1 and March 30, 2020. Serum concentrations of PGRN were determined by enzyme-linked immunosorbent assay in patients with COVID-19 at hospital admission and at discharge. In parallel, the serum levels of soluble adhesion molecules, vascular cell adhesion molecule-1 (sVCAM-1), intercellular adhesion molecule-1 (sICAM1), P-selectin (sP-selectin), and E-selectin (sE-selectin) were assayed by a human adhesion molecule multiplex kit. The association between serum PGRN levels and other laboratory test results was analyzed by Spearman correlation analysis.

Results: At baseline, the median serum PGRN levels in patients with COVID-19 were $94.8 \mathrm{ng} /$ $\mathrm{mL}$ [interquartile range (IQR): $66.6-119.6 \mathrm{ng} / \mathrm{mL}$ ], which was significantly elevated compared with those in healthy controls $(46.3 \mathrm{ng} / \mathrm{mL}$, IQR: $41.8-55.6 \mathrm{ng} / \mathrm{mL})$. Moreover, the median serum sVCAM-1 levels were significantly higher in COVID-19 patients (1396.0 ng/mL, IQR: $1019.1-1774.8 \mathrm{ng} / \mathrm{mL})$ than those in healthy controls $(612.4 \mathrm{ng} / \mathrm{mL}$, IQR: $466.4-689.3 \mathrm{ng} / \mathrm{mL})$. However, the levels of sICAM-1, sP-selectin, and sE-selectin were not significantly elevated in patients with COVID-19 when compared to healthy controls. Further analysis showed that serum PGRN levels were significantly positively associated with sVCAM-1 $(\mathrm{r}=0.675, P=0.008)$ and inversely with sICAM-1 $(\mathrm{r}=-0.609, P=0.021)$ and aspartate aminotransferase levels $(\mathrm{r}=-0.560$, $P=0.037$ ) in patients with COVID-19 at hospital admission. In COVID-19 patients, serum PGRN and sVCAM-1 levels fell significantly after successful treatment.

Conclusion: The present study demonstrates elevated serum PGRN and sVCAM-1 levels in patients with COVID-19, which may provide clues as to the mechanisms underlying the pathogenesis of COVID-19. Further studies are warranted to evaluate the potential of PGRN and sVCAM-1 as biomarkers and investigate their role in the pathogenesis of COVID-19.

Keywords: COVID-19, PGRN, soluble adhesion molecules, pathogenesis, biomarker

\section{Introduction}

Coronavirus disease 2019 (COVID-19) is a well-known contagious disease caused by severe acute respiratory syndrome coronavirus 2 (SARS-CoV-2), which has so far killed millions of lives worldwide. According to the data released by the World Health Organization, ${ }^{1}$ as of July 19, 2021, more than 188 million cases have been confirmed and over 4 million cases have died of SARS-CoV-2, and more alarming is that the number of the cases is still on the increase. 
Evidence has shown that COVID-19 patients, especially those severe and critical patients, commonly had histological characteristics of endotheliopathy, vascular thrombosis, and new vessel growth in lungs, ${ }^{2-6}$ suggesting the presence of endothelial activation and recruitment of immune cells as well as angiogenesis in the lesions caused by SARS-CoV-2 infection. To understand the immunology of SARS-CoV-2 infection, efforts had been made to reveal differentially regulated markers in COVID-19 patients, ${ }^{7-9}$ and dozens of molecules, including endothelial cell adhesion molecules and matrix metalloproteinase-1 (MMP-1), have been successively identified. Nonetheless, the pathophysiological factors that contribute to the pathogenesis of COVID-19 remain inadequately defined.

Progranulin (PGRN) is a cysteine-rich glycoprotein, which can be secreted by endothelial cells and immune cells, such as macrophage following multiple infections. ${ }^{10,11}$ PGRN mediates a variety of biological functions, including host defense, inflammation, and tumorigenesis. ${ }^{10,12,13}$ Cross-sectional studies have shown that serum PGRN levels were increased in patients with human immunodeficiency virus (HIV) and hepatitis $\mathrm{B}$ virus (HBV) infection. ${ }^{12,14}$ Animal experiments confirmed the observation that PGRN was up-regulated in infection models and revealed that PGRN was implicated in host defense against bacterial pneumonia, influenza virus-induced lung injury, and sepsis. ${ }^{10,11,15}$ However, whether serum PGRN levels are quantitatively elevated in COVID-19 patients remain to be determined.

Growing evidence shows that the activated endothelial cells play a pivotal role in the progression of SARS-CoV -2-induced inflammation and suggests that the levels of serum soluble endothelial adhesion makers, such as sVCAM-1, sICAM-1, sP-selectin, are associated with disease severity of COVID-19. ${ }^{6,8}$ However, discrepant results regarding the levels of sVCAM-1 and sICAM-1 have been observed between two cohorts of patients with mild/moderate COVID-19. ${ }^{8,9}$ E-selectin is another important inducible endothelial cell surface molecule, which can also be transcriptionally upregulated by proinflammatory factors, such as tumor necrosis factor- $\alpha$ (TNF- $\alpha$ ) and interleukin 1 (IL-1), and hence mediates rolling of leukocytes, such as neutrophils, lymphocytes and monocytes on the endothelium. ${ }^{16}$ No study has investigated the levels of sEselectin in patients with COVID-19.

Therefore, the objective of this study was to quantitatively determine the serum levels of PGRN and the soluble adhesion molecules, sVCAM-1, sICAM-1, sP-selectin and
sE-selectin in patients with COVID-19, as well as to evaluate whether serum PGRN levels correlate to endothelial activation markers and other laboratory test results.

\section{Methods}

\section{Participants}

In this cross-sectional study, fourteen laboratory-confirmed COVID-19 cases from the Zunyi Medical University Third Affiliated Hospital $(n=12)$ and the Fourth People's Hospital of Zunyi $(n=2)$ between 1 February and 30 March, 2020, were enrolled. The diagnosis of COVID-19 was made according to the guideline for COVID-19, which was officially issued by the National Health Commission of the People's Republic of China. ${ }^{17}$ Mild/moderate cases were defined as patients having lesions in lungs visible on high-resolution computed tomography images and positive nucleic acid tests for SARS$\mathrm{CoV}-2$. Severe cases were patients had one or more of the following evidence, including respiratory distress with a respiratory rate more than 30 times per minute, oxygen saturation less than $93 \%$ at rest, oxygenation index less than $300 \mathrm{mmHg}$, and respiratory failure requiring ventilation. All patients received drugs of interferon $\alpha-2 b$, lopinavir, and Chinese medicine as suggested by the guideline. Patients were discharged when their clinical signs and symptoms were effectively improved, and especially when they had two consecutive SARS-CoV-2 nucleic acid tests (24-hours apart) negative. ${ }^{17}$

Patient characteristics including age, sex, disease history, laboratory test results, and treatments were retrieved from hospital information system and laboratory information system. To have reference ranges of serum PGRN and soluble adhesion molecules, we enrolled additional fourteen volunteers who went to the First People's Hospital of Zunyi for annual healthy checkup between August 25 and August 28, 2020. These healthy subjects were not previously or currently infected with SARS-CoV-2.

\section{Sample Collection and Storage}

For patients, blood samples were collected on the day of hospital admission or hospital discharge, and fasting blood samples were drawn for testing of blood glucose (Glu), urea nitrogen (BUN), PGRN and soluble adhesion molecules. For healthy donors, fasting blood samples were collected on the morning of the checkup day. The remaining serum samples were safely kept at $-80^{\circ} \mathrm{C}$ prior to further testing. 


\section{Laboratory Testing}

The China National Accreditation Service for Conformity Assessment (CNAS) has accredited the laboratory testing at the department of laboratory medicine of the First People's Hospital of Zunyi under the ISO15189 accreditation standard. Routine blood tests, including whole-blood cell counts, lymphocyte counts, neutrophil counts, monocyte counts were analyzed on the Sysmex XN-1000 Hematology Analyzer. C-reactive protein (CRP) was determined by the turbidimetry method on the Lifotronic PA900 analyzer (Shenzhen, China). Biochemical analytes including alanine aminotransferase (ALT), aspartate aminotransferase (AST), BUN, Glu, were determined on Beckman coulter AU5800 automatic biochemistry analyzer.

The presence of SARS-CoV-2 RNA on nasopharyngeal swabs was detected using two different real-time PCR kits provided by Zhongshan Daan gene Biotech. Co., Guangzhou, China, and Shanghai Zhijiang Biotech. Co., Shanghai, China, and run on the SLAN96 real-time PCR instrument (Shanghai Hongshi Biotechnology Co., Shanghai, China). Anti-HIV antibodies were detected by chemiluminescence assay on the Autobio A2000 plus analyzer (Zhengzhou, China). Influenza A virus (Flu A) IgM, influenza B virus (Flu B) IgM, Mycoplasma pneumoniae (M. pneumoniae) IgM, parainfluenza (PIV) IgM, adenovirus IgM, respiratory syncytial virus (RSV) IgM, Legionella pneumophila (L. pneumophila) IgM, Chlamydia pneumoniae IgM, and Rickettsiae IgM were detected by indirect immunofluorescence assay (Nine respiratory tract pathogens IgM detection kit; Vircell Inc., Spain).

\section{Luminex Assay and Enzyme-Linked Immunosorbent Assay}

Levels of sVCAM-1, sICAM-1, sP-selectin, and sE-selectin were determined using Magnetic Luminex High Performance Assay kits (Catalog number: LKTM007, R\&D Systems, USA) and analyzed on the Magpix platform according to the manufacturer's instructions. PGRN levels were determined using commercially available ELISA kit (R\&D Systems, USA) according to the manufacturer's instructions.

\section{Statistical Analysis}

Data were analyzed using IBM SPSS V.19.0 software (IBM Corp, Armonk, NY, USA) and further processed, where necessary, using GraphPad Prism 5.0 or Microsoft Excel 2007. All categorical variables were reported as frequency and percentage, and their differences among the groups were compared using the Pearson $\chi^{2}$ or Fisher's exact test. Quantitative data were reported as median with interquartile range (IQR) and were compared between the groups using the Mann-Whitney test. The association between PGRN and other laboratory index data was analyzed using the Spearman's rank correlation test. Levels of PGRN and soluble adhesion molecules in patients at the onset of illness and on the day of discharge were compared by using Wilcoxon signed-rank test. $P$ value $<0.05$ was considered as statistically significant.

\section{Results}

\section{Characteristics of Enrolled Subjects}

Overall, fourteen COVID-19 patients, and fourteen healthy controls were included in this study. Their demographic data and clinical characteristics are summarized in Table 1. Briefly, both groups were equally with seven males and seven females. The median ages were 40 years (IQR: $18.3-53.0$ ) and 36 years (IQR: 27.8-45.8) for the patients and the healthy controls, respectively. All healthy controls were negative for SARSCoV-2 infection. Significant differences in age, gender, disease history, eg, diabetes mellitus (DM) and chronic hepatitis disease between patients and healthy controls were not found $(P>$ $0.05)$. All patients and healthy controls were tested negative for anti-HIV antibodies. The median serum levels of ALT ( $33.3 \mathrm{U} /$ L vs $13.4 \mathrm{U} / \mathrm{L}, P<0.001)$, AST (34.9 U/L vs $18.9 \mathrm{U} / \mathrm{L}, P<$ $0.001)$, and fasting blood glucose $(6.1 \mathrm{mmol} / \mathrm{L}$ vs $4.7 \mathrm{mmol} / \mathrm{L}$, $P<0.001)$ were significantly higher in COVID-19 patients than healthy controls, respectively. Lymphocyte counts and the levels of BUN in COVID-19 patients were significantly lower than those in healthy controls, while significant differences in whole-blood cell counts, neutrophil counts, monocyte counts and levels of CRP were not found between groups. Of the twelve cases with serological test results, it was found that six $(50 \%)$ were positive for other viral or atypical bacterial IgM antibodies. Specifically, three, one, two and two patients were M. pneumoniae IgM, C. pneumoniae IgM, Parainfluenza IgM, and Influenza B virus IgM positive, respectively, one was both M. pneumoniae and Parainfluenza IgM positive, and one was both M. pneumoniae and Influenza B virus IgM positive.

\section{PGRN and Soluble Adhesion Molecules Levels in COVID-19 Patients and Health Controls}

The median serum levels of PGRN in COVID-19 patients were significantly higher at $94.8 \mathrm{ng} / \mathrm{mL}$ (IQR: $66.6-119.6$ $\mathrm{ng} / \mathrm{mL})$ than those in healthy controls $(46.3 \mathrm{ng} / \mathrm{mL}, \mathrm{IQR}$ : 
Table I Demographic and Clinical Characteristics of the Enrolled Subjects

\begin{tabular}{|c|c|c|c|}
\hline Characteristics & COVID-19 Patients $(n=14)$ & HC $(n=14)$ & $P$ value \\
\hline Age, years, median (range) & $40(18.3-53.0)$ & $36(27.8-45.8)$ & 0.945 \\
\hline Male/female (n) & $7 / 7$ & $7 / 7$ & \\
\hline Smoking & $2 / 14(14.3 \%)$ & $0 / 14(0 \%)$ & \\
\hline Alcohol usage & $2 / 14(14.3 \%)$ & $2 / 14(14.3 \%)$ & \\
\hline Diabetes mellitus & $1 / 14(7.1 \%)$ & $0 / 14(0 \%)$ & \\
\hline Cardiovascular disease & $1 / 14(7.1 \%)$ & $0 / 14(0 \%)$ & \\
\hline Autoimmune disease & $0 / 14(0 \%)$ & $0 / 14(0 \%)$ & \\
\hline Chronic hepatitis disease & $0 / 14(0 \%)$ & $0 / 14(0 \%)$ & \\
\hline ALT (U/L) & $33.3(20.5-56.9)$ & $13.4(\mid 0.7-15.6)$ & $<0.001$ \\
\hline AST (U/L) & $34.9(28.3-39.4)$ & $18.9(\mid 7.6-22.3)$ & $<0.001$ \\
\hline BUN (mmol/L) & $3.6(3.0-5.1)$ & $4.4(4.0-5.3)$ & 0.048 \\
\hline Glu (mmol/L) & $6.1(5.4-6.7)$ & $4.7(4.3-5.1)$ & $<0.001$ \\
\hline Whole blood cell count $\left(\times 10^{9} / \mathrm{L}\right)$ & $5.4(4.2-6.6)$ & $6.2(4.9-6.7)$ & 0.421 \\
\hline Neutrophil count $\left(\times 10^{9} / \mathrm{L}\right)$ & $3.4(2.3-4.3)$ & $3.9(2.4-4.2)$ & 0.765 \\
\hline Lymphocyte count $\left(\times 10^{9} / \mathrm{L}\right)$ & $1.3(1.0-1.8)$ & $1.9(1.7-2.1)$ & 0.025 \\
\hline Monocyte count $\left(\times 10^{9} / \mathrm{L}\right)$ & $0.5(0.3-0.7)$ & $0.5(0.3-0.5)$ & 0.214 \\
\hline CRP (mg/L) & $0.9(0.5-5.5)$ & $0.9(0.6-1.9)$ & 0.547 \\
\hline Duration from symptom onset to admission, median (IQR), days & I (0-7) & l & \\
\hline Anti-HIV antibodies & $0 / 14(0 \%)$ & $0 / 14(0 \%)$ & \\
\hline M. pneumoniae IgM & $3 / 12(25.0 \%)$ & NA & \\
\hline C. pneumoniae IgM & $1 / 12(8.3 \%)$ & NA & \\
\hline Parainfluenza IgM & $2 / 12(16.7 \%)$ & NA & \\
\hline Influenza $B$ virus $\lg M$ & $2 / 12(16.7 \%)$ & NA & \\
\hline Severe case & $1 / 14$ (7.1\%) & I & \\
\hline
\end{tabular}

Notes: Data are median (IQR) or $\mathrm{n} / \mathrm{N}(\%)$, where $\mathrm{N}$ is the number of patients with available data; /, not applicable; $P$ values were determined by Mann-Whitney $U$-test. Abbreviation: NA, not available.

41.8-55.6 $\mathrm{ng} / \mathrm{mL}$ ) as shown in Figure 1. The median serum levels of sVCAM-1 in COVID-19 patients were $1396.0 \mathrm{ng} / \mathrm{mL}$ (IQR: 1019.1-1774.8 ng/mL), which was also significantly higher than those in healthy controls (612.4 $\mathrm{ng} / \mathrm{mL}$, IQR: $466.4-689.3 \mathrm{ng} / \mathrm{mL}$ ). However, the median serum levels for other adhesion molecules were $80.8 \mathrm{ng} / \mathrm{mL}$ (sICAM-1), $54.3 \mathrm{ng} / \mathrm{mL}$ (sP-selectin), and $23.0 \mathrm{ng} / \mathrm{mL}$ (sE-selectin) in COVID-19 patients, which were not significantly higher than those in healthy controls at $66.4 \mathrm{ng} / \mathrm{mL}$ (sICAM-1), $57.0 \mathrm{ng} / \mathrm{mL}$ (sP-selectin), and $19.2 \mathrm{ng} / \mathrm{mL}$ (sE-selectin), respectively (Figure 1).

\section{Association of Serum PGRN with Available Laboratory Test Results in COVID-19 Patients}

The serum levels of PGRN were positively correlated with those of sVCAM-1 in COVID-19 patients at the time of admission (Figure 2). Furthermore, serum PGRN levels were inversely correlated with the levels of sICAM-1 and AST in patients (Figure 2). No correlation was noted between serum levels of PGRN and sP- selectin as well as sE-selectin (Figure S1). Moreover, no correlation was found between serum PGRN and other routine laboratory tests, including CRP, glucose, and lactate dehydrogenase (LDH) in COVID-19 patients (Figure S2).

\section{Reduced Levels of PGRN and sVCAM-I Following Effective Therapy}

Five patients were lost to follow-up, because they were moved to the provincial designated hospital for further treatment; thus, nine patients were recruited on the day of hospital discharge (median hospitalization days: 13, IQR: 8-20), and serum levels of PGRN and soluble adhesion molecules were determined. Both serum levels of PGRN and sVCAM-1 were significantly decreased in the patients who have recovered from COVID-19, when compared with their corresponding baseline levels (Figure 3). Significant differences were not observed for the other adhesion molecules, sICAM-1, sPselectin, and sE-selectin. 
A

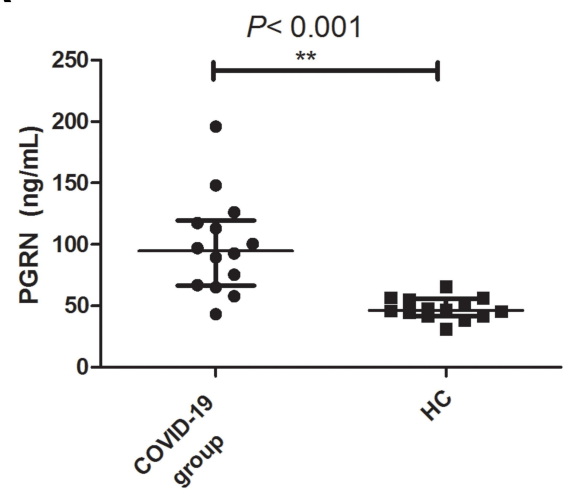

C

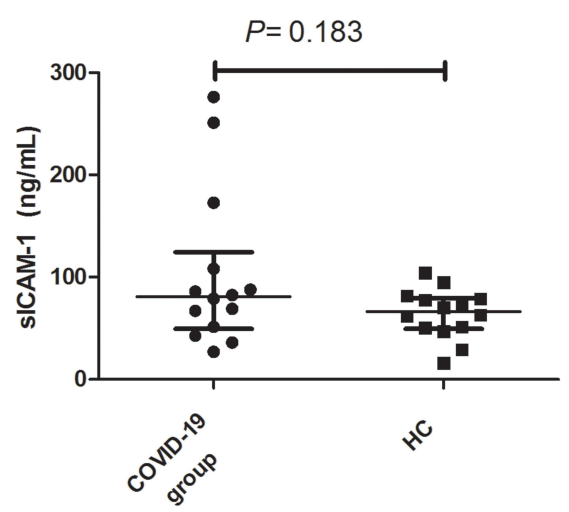

E

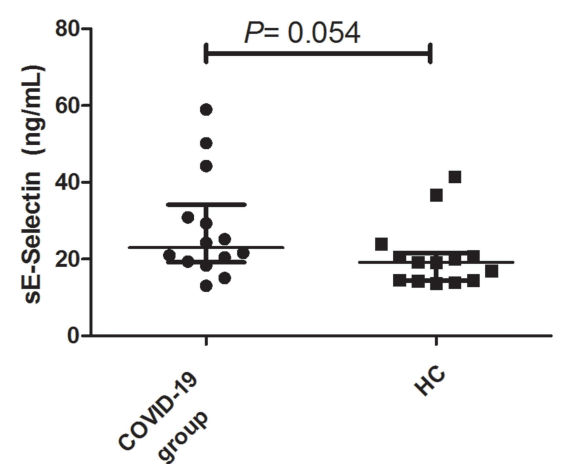

B

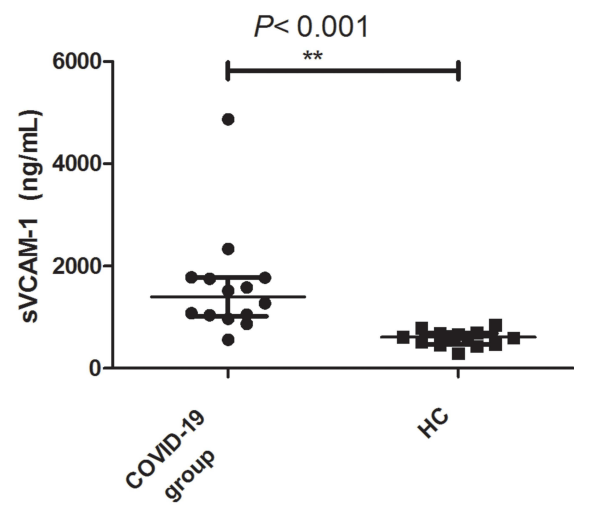

D

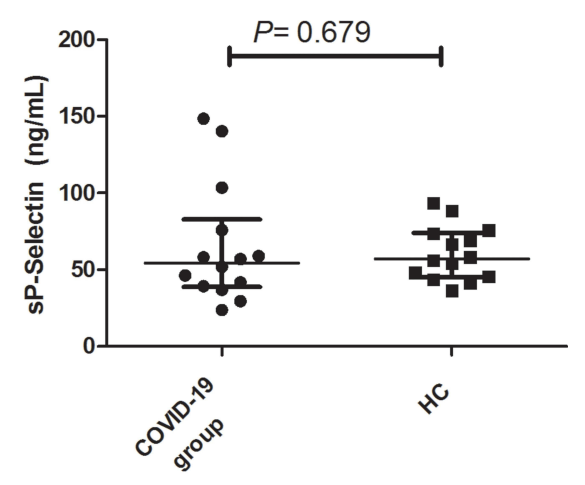

Figure I Scatter-plots of serum levels of PGRN, and soluble adhesion molecules in COVID-19 patients on admission and healthy controls (HC). Serum levels of PGRN (A) were determined using an ELISA assay kit, and serum levels of sVCAM-I (B), sICAM-I (C), sP-Selectin (D), sE-Selectin (E) were determined using the Luminex assay kit designed for soluble adhesion molecules. The horizontal lines represent the median concentrations of the indicated indexes in both groups. $P$ values are indicated in the figures, and all comparisons were conducted using Mann Whitney U-test.

\section{Discussion}

Lymphocytic inflammation, microvascular injury, and new vessel growth have been recognized as hallmarks of the pathology in the lungs of patients with COVID-19. ${ }^{5,6} \mathrm{We}$ show here that serum levels of PGRN and SVCAM-1, but not sICAM-1, sP-selectin and sE-selectin, are significantly increased and related to response to therapy in patients with COVID-19. Moreover, we demonstrate that serum levels of PGRN positively correlate with the levels of endothelial activation marker of sVCAM-1 and inversely correlate with the levels of sICAM-1 and AST. Our data are in line with a very recent report on the levels of PGRN in COVID-19, which was based on proximity extension assay but did not quantitatively determine the levels of PGRN. ${ }^{18}$ Moreover, our data are not completely in agreement with previous results from Tong et $\mathrm{al}^{8}$ and Syed 
A

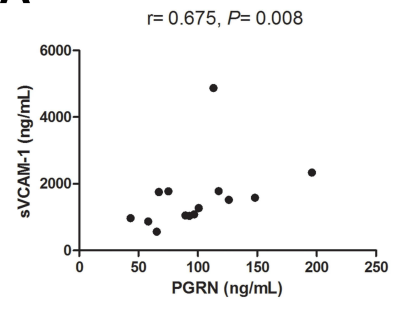

B

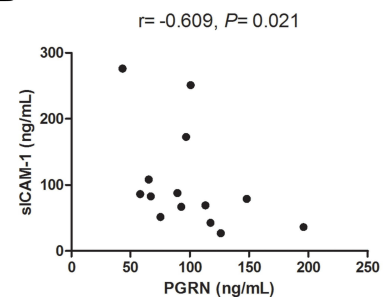

C

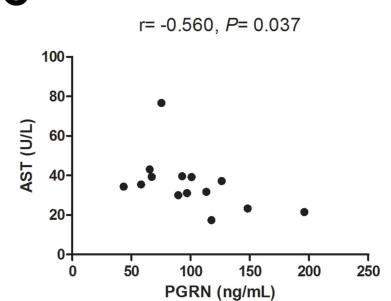

Figure 2 Correlations of serum PGRN levels with other laboratory test results in patients with COVID-19 on admission. (A) Spearman correlation analysis between serum levels of PGRN and sVCAM-I in COVID-19 patients. (B) Spearman correlation analysis between serum levels of PGRN and sICAM-I in COVID-19 patients. (C) Spearman correlation analysis between serum levels of PGRN and AST in COVID-19 patients. $r$ and $P$ values are indicated in the figures.

et al, ${ }^{9}$ showing significantly increased levels of sVCAM-1 in patients with mild/moderate COVID-19, which probably results from the varied analytical performance of the assay kits used and differences in the severity of patients. Finally, for the first time, we investigated the levels of sEselectin in patients with COVID-19.

There is evidence showing an impaired antiviral immune response in COVID-19. ${ }^{19}$ Interferons play critical roles in the antiviral immune response as they harbor capacity to reduce viral replication; however, a repression of type I interferon signaling has been observed in patients with COVID-19. ${ }^{19,20}$ It is known from the influenza virus infection that PGRN is involved in the attenuation of type I interferon signaling, which impairs the survival of mice from lethal infection. ${ }^{10,13}$ The significant increase in levels of PGRN in COVID-19 patients may therefore suggest that the PGRN-mediated antiviral strategy is also functional in SARS-CoV-2 infection. Nevertheless, Thurner et $\mathrm{al}^{21}$ have recently reported the presence of autoantibodies against PGRN in patients with critical COVID-19 and showed that plasma from PGRN-Autoantibodies-negative patients was more effective than plasma from PGRN-Autoantibodies-positive patients to neutralize the TNF- $\alpha$-induced cytotoxicity. It was therefore proposed that the significantly decreased levels of PGRN are responsible for the critical COVID19. Based on these conflicting observations, further studies are warranted to address the potential roles of PGRN in the pathogenesis of COVID-19. In addition, since convalescent plasma therapy is suggested for use in patients with critical COVID-19, ${ }^{22}$ quantitative determination of PGRN levels is therefore required, and it seems that only plasma with PGRN in normal ranges is acceptable.

SARS-CoV-2 infection is histologically featured by angiocentric inflammation with endothelial injury and massive leukocyte infiltration, as well as thrombosis in some severe cases. ${ }^{6}$ Since VCAM-1 is normally absent on resting endothelial cells but could be significantly induced following TNF- $\alpha$ and IL-1 stimulation, ${ }^{23}$ the high levels of sVCAM-1 in patients with COVID-19 therefore indicate the presence of endothelial activation and leukocyte infiltration. It's known that VCAM-1 signaling is involved in the activation of endothelial cell-associated matrix metalloproteinases (MMPs), which digest the extracellular matrix, and is implicated in the activation of endothelial cell NADPH dual oxidase 2 (NOX2) which leads to the formation of intercellular gap, suggesting the critical role of VCAM signaling in thrombosis by exposing tissue factors. Moreover, it has been reported that sVCAM-1 have inhibitory effects on $\mathrm{T}$ cell activation in rheumatoid arthritis, ${ }^{24}$ however, whether this effect occurs in the pathogenesis of COVID-19 is worthy of further investigation. Even with obvious infection lesions in lungs of patients enrolled in this cohort, the levels of CRP were not significantly higher in patients as compared to healthy controls, which is consistent with the result from a previous meta-analysis showing that CRP is only robustly elevated in severe/critical cases. ${ }^{25}$ Additionally, it is known that PGRN could competitively bind to tumor necrosis factor receptor 1 (TNFR1) 26,27 and TNF- $\alpha$ / TNFR1 is implicated in activating coagulation pathways, ${ }^{28}$ it is therefore possible that the high levels of PGRN may block TNF- $\alpha$ mediated inflammation and probably coagulation pathways in COVID-19. Presumably, a lack of competitive blockage of TNF- $\alpha$ induced coagulatory response due to decreased levels of PGRN in patients with severe/critical COVID-19 may be one of the mechanisms connecting PGRN to the disease severity of COVID-19 as discussed above.

SARS-CoV-2 infection is associated with angiogenesis. Ackermann et al reported the formation of new blood vessels in lungs of patients infected with SARS-CoV-2 at 
A

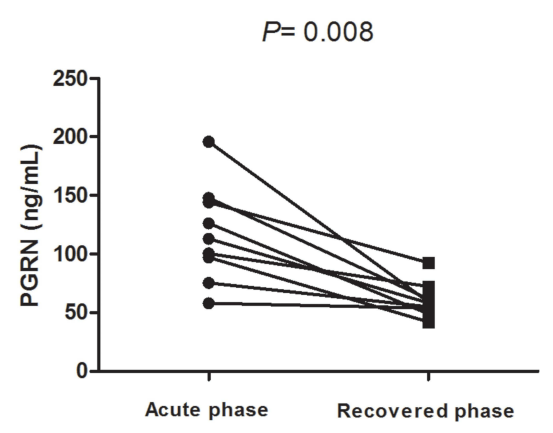

C

$P=0.515$

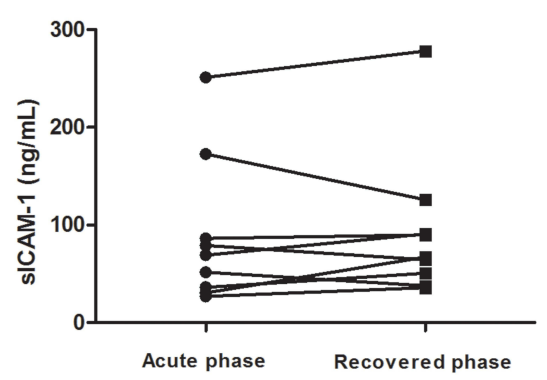

E

$P=0.139$

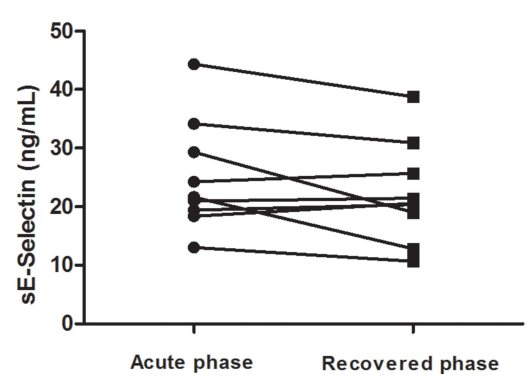

B

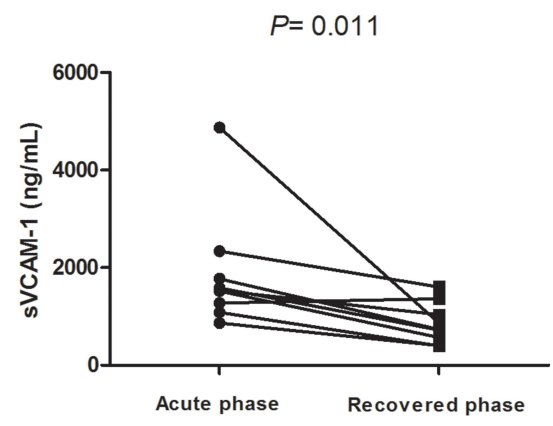

D

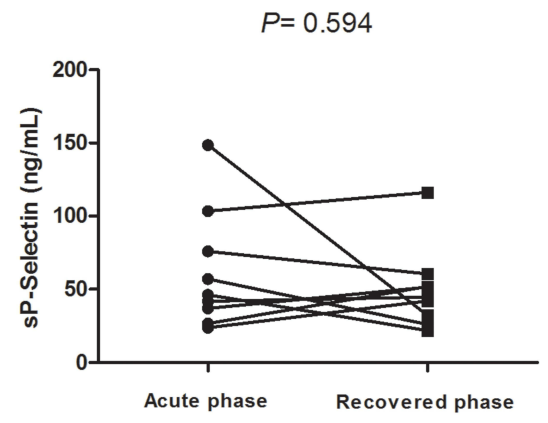

Figure 3 Serum levels of PGRN and sVCAM-I in patients with COVID-I9 were decreased following effective therapy. Serum levels of PGRN (A), sVCAM-I (B), sICAM-I (C), sP-selectin (D), and sE-selectin (E) in COVID-19 patients on hospital admission (acute phase) and discharge (recovered phase) were determined and compared. Wilcoxon signed-rank test was used to assess the differences. $P$ values are indicated in the figures.

autopsy. ${ }^{5}$ The mechanisms underlying angiogenesis in COVID-19 remain to be determined. Tan et $\mathrm{al}^{29}$ reported on elevated angiogenic cytokines, eg, vascular endothelial growth factor (VEGF) and hepatocyte growth factor (HGF) in COVID-19 patients. Other factors, including PGRN, VCAM-1, and trace elements, such as copper, are also found to have a profound impact on angiogenesis. ${ }^{30-32} \mathrm{We}$ show here that both PGRN and sVCAM-1 are elevated in
COVID-19 patients, suggested a potential involvement of PGRN and sVCAM-1 in the formation of new vessels in COVID-19. Although the present study demonstrates that the levels of PGRN correlate positively with the levels of sVCAM-1, it remains unclear whether those factors have any direct and collaborative effect on the angiogenesis in COVID-19. Experimental studies are required to address this question. 
Apart from the acute effects, SARS-CoV-2 infection has been associated with long-term damage to organs, particularly the heart. ${ }^{33,34}$ A previous study of 100 German patients with COVID-19 showed that $60 \%$ of the cases had cardiac injuries even after recovery. ${ }^{34}$ The underlying mechanisms remain obscure. However, considering PGRN is implicated in inhibition of NF- $\mathrm{KB}$ signaling-mediated inflammation, evidence from Thurner et $\mathrm{al}^{21}$ may suggest that the long-term cardiac injuries are possibly associated with significantly decreased levels of PGRN due to the presence of PGRN autoantibodies. In addition, Pour et $\mathrm{al}^{35}$ reported on significantly decreased levels of zinc in Iranian patients with critical COVID-19 and demonstrated that low concentrations of zinc were associated with poor outcomes of the disease. This finding may suggest an impaired role for zinc in the modulation of NF$\kappa \mathrm{B}$ signaling and redox signaling, as well as direct inhibition of viral entry and replication in COVID-19, as proposed elsewhere. ${ }^{36,37}$ Together, these observations may suggest the importance of monitoring levels of endothelial activation markers, such as sVCAM-1, trace elements, such as zinc, and the proinflammatory factors, such as TNF- $\alpha$ in patients even recovered from SARSCoV-2 infection.

It is well known that ICAM-1 also plays an important role in mediating the recruitment of leukocytes from circulation to sites of inflammation. ${ }^{38}$ In line with previous findings, ${ }^{4,8}$ the present work shows that serum levels of sICAM-1 are elevated in patients with COVID-19 as compared to healthy control, albeit not significantly higher due to small patient numbers. We show here that serum PGRN levels inversely correlate with the levels of ICAM1 in patients with COVID-19. The reasons for this observation remain obscure in the current situation. One possible explanation would be that PGRN is able to inhibit the production of ICAM-1, because it has been shown that exogenous PGRN reduces the expression of ICAM-1 in human umbilical venous endothelial cells. ${ }^{39}$ The regulatory mechanisms of ICAM-1 by PGRN and its implication in the pathogenesis of COVID-19 remain to be determined.

Evidence indicates an increase of P-selectin on the surface of platelets following SARS-CoV-2 infection. ${ }^{40}$ Nevertheless, the present study did not show significant differences in levels of sP-selectin between the two groups, which is coincident with the finding from Goshua et al. ${ }^{6}$ While P-selectin blockade may be helpful to alleviate the pathobiology of acute respiratory distress syndrome (ARDS) in patients with severe/critical COVID-19, ${ }^{41}$ the present evidence does not support preventive usage of a P-selectin inhibitor in patients with mild/moderate COVID-19 to avoid thrombosis or ARDS.

The major limitation of this study is the small sample size and heterogeneity of the patients enrolled, so that some data may not be effectively analyzed, for instance, the significance of correlations between the variables. Second, the majority of the patients were mild/moderate cases that may not reflect the full picture of the patients with COVID-19. Third, the impact of co-infection with other organisms on the levels of serum PGRN and soluble adhesion molecules was not evaluated due to the small number of the cases.

In conclusion, although the sample size is relatively small, the present study shows elevated serum levels of both PGRN and sVCAM-1 in patients with COVID-19, suggesting their potential as biomarkers and roles in the pathogenesis of COVID-19. Further studies are warranted to strengthen these findings and investigate the potential roles and mechanisms of PGRN and sVCAM-1 in the pathogenesis of COVID-19.

\section{Ethics Statement}

This study was in compliance with the Helsinki Declaration and was approved by the Human Research Ethics Committee of the First People's Hospital of Zunyi (Registration number: 2020-03, Approved on 12 Mar, 2020). Since any nonmedical contact with the patients or their lineal relatives is prohibited according to the institutional requirements for clinical management of COIVD-19 and the use of surplus clinical blood samples does not cause direct patient harm, a waiver of informed consent from patients was approved by the Human Research Ethics Committee of the First People's Hospital of Zunyi. Signed consents were obtained from healthy subjects before sample collection.

\section{Funding}

This study was supported by Cooperation Project provided by Zunyi city and the First People's Hospital of Zunyi (HZzi-2020-2), Science and Technology Project of Guizhou Health Commission (gzwjkj2019-1-113), Novel Coronavirus Special Research Project provided by Science and Technology Department of Guizhou Province (QianKehe-ZhiCheng [2020]4Y201), and Innovation Group Project provided by Education Department of Guizhou Province (QianJiaoheKYzi [2021]019). 


\section{Disclosure}

The authors report no conflicts of interest in this work.

\section{References}

1. World Health Organization. WHO coronavirus (COVID-19) dashboard; 2021. Available from: www.who.int/emergencies/dis eases/novel-coronavirus-2019. Accessed July 19, 2021.

2. Hariri L, Hardin CC. COVID-19, angiogenesis, and ARDS endotypes. $N$ Engl J Med. 2020;383(2):182-183. doi:10.1056/NEJMe 2018629

3. Lowenstein CJ, Solomon SD. Severe COVID-19 is a microvascular disease. Circulation. 2020;142(17):1609-1611. doi:10.1161/ CIRCULATIONAHA.120.050354

4. Nagashima S, Mendes MC, Camargo Martins AP, et al. Endothelial dysfunction and thrombosis in patients with COVID-19-brief report. Arterioscler Thromb Vasc Biol. 2020;40(10):2404-2407. doi:10.1161/ ATVBAHA.120.314860

5. Ackermann M, Verleden SE, Kuehnel M, et al. Pulmonary vascular endothelialitis, thrombosis, and angiogenesis in COVID-19. $N$ Engl $J$ Med. 2020;383(2):120-128. doi:10.1056/NEJMoa2015432

6. Goshua G, Pine AB, Meizlish ML, et al. Endotheliopathy in COVID-19-associated coagulopathy: evidence from a single-centre, cross-sectional study. Lancet Haematol. 2020;7(8):e575-e582. doi:10.1016/S2352-3026(20)30216-7

7. Li L, Huang M, Shen J, et al. Serum levels of soluble platelet endothelial cell adhesion molecule 1 in COVID-19 patients are associated with disease severity. $J$ Infect Dis. 2021;223(1):178-179. doi:10.1093/infdis/jiaa642

8. Tong M, Jiang Y, Xia D, et al. Elevated expression of serum endothelial cell adhesion molecules in COVID-19 patients. J Infect Dis. 2020;222(6):894-898. doi:10.1093/infdis/jiaa349

9. Syed F, Li W, Relich RF, et al. Excessive matrix metalloproteinase-1 and hyperactivation of endothelial cells occurred in COVID-19 patients and were associated with the severity of COVID-19. J Infect Dis. 2021;224(1):60-69. doi:10.1093/infdis/jiab167

10. Luo Q, Yan X, Tu H, Yin Y, Cao J. Progranulin aggravates pulmonary immunopathology during influenza virus infection. Thorax. 2019;74(3):305-308. doi:10.1136/thoraxjnl-2018-211916

11. Zou S, Luo Q, Song Z, et al. Contribution of progranulin to protective lung immunity during bacterial pneumonia. $J$ Infect Dis. 2017;215 (11):1764-1773. doi:10.1093/infdis/jix197

12. Gong Y, Zhan T, Li Q, et al. Serum progranulin levels are elevated in patients with chronic hepatitis B virus infection, reflecting viral load. Cytokine. 2016;85:26-29. doi:10.1016/j.cyto.2016.05.026

13. Wei F, Jiang Z, Sun H, et al. Induction of PGRN by influenza virus inhibits the antiviral immune responses through downregulation of type I interferons signaling. PLoS Pathog. 2019;15(10):e1008062. doi:10.1371/journal.ppat.1008062

14. Suh HS, Lo Y, Choi N, Letendre S, Lee SC. Evidence of the innate antiviral and neuroprotective properties of progranulin. PLoS One. 2014;9(5):e98184. doi:10.1371/journal.pone.0098184

15. Song Z, Zhang X, Zhang L, et al. Progranulin plays a central role in host defense during sepsis by promoting macrophage recruitment. $\mathrm{Am}$ J Respir Crit Care Med. 2016;194(10):1219-1232. doi:10.1164/ rccm.201601-0056OC

16. Wang Y, Xu Y, Yang M, Zhang M, Xiao M, Li X. Butyrate mitigates TNF- $\alpha$-induced attachment of monocytes to endothelial cells. $J$ Bioenerg Biomembr. 2020;52(4):247-256. doi:10.1007/s10863020-09841-9

17. National Health Commission of the People's Republic of China. Clinical protocols for COVID-19 (The modified fifth and the sixth editions). Available from: http://www.nhc.gov.cn/xcs/zhengcwj/list_ gzbd_6.shtml. Accessed February 8, 2020.
18. Rieder M, Wirth L, Pollmeier L, et al. Serum protein profiling reveals a specific upregulation of the immunomodulatory protein progranulin in coronavirus disease 2019. J Infect Dis. 2020;223(5):775-784. doi:10.1093/infdis/jiaa741

19. Vanderheiden A, Ralfs P, Chirkova T, et al. Type I and type III interferons restrict SARS-CoV-2 infection of human airway epithelial cultures. J Virol. 2020;94(19):e00985-20. doi:10.1128/JVI.00985-20

20. McNab F, Mayer-Barber K, Sher A, Wack A, O'Garra A. Type I interferons in infectious disease. Nat Rev Immunol. 2015;15 (2):87-103. doi:10.1038/nri3787

21. Thurner L, Fadle N, Bewarder M, et al. Autoantibodies against progranulin and IL-1 receptor antagonist in critically ill COVID-19. bioRxiv. 2021:441188. doi:10.1101/2021.04.23.441188

22. Brown BL, McCullough J. Treatment for emerging viruses: convalescent plasma and COVID-19. Transfus Apher Sci. 2020;59(3):20. doi:10.1016/j.transci.2020.102790

23. Kong DH, Kim YK, Kim MR, Jang JH, Lee S. Emerging roles of vascular cell adhesion molecule-1 (VCAM-1) in immunological disorders and cancer. Int J Mol Sci. 2018;19(4):1057. doi:10.3390/ ijms 19041057

24. Kitani A, Nakashima N, Matsuda T, et al. T cells bound by vascular cell adhesion molecule-1/CD106 in synovial fluid in rheumatoid arthritis: inhibitory role of soluble vascular cell adhesion molecule-1 in T cell activation. J Immunol. 1996;156(6):2300-2308.

25. Hariyanto TI, Japar KV, Kwenandar F, et al. Inflammatory and hematologic markers as predictors of severe outcomes in COVID-19 infection: a systematic review and meta-analysis. $\mathrm{Am}$ J Emerg Med. 2021;41:110-119. doi:10.1016/j.ajem.2020.12.076

26. Kotela A, Wojdasiewicz P, Łęgosz P, et al. Increased serum levels of progranulin (PGRN) in patients with haemophilic arthropathy. Clin Exp Pharmacol Physiol. 2019;46(4):373-379. doi:10.1111/14401681.13054

27. Jian J, Konopka J, Liu C. Insights into the role of progranulin in immunity, infection, and inflammation. J Leukoc Biol. 2013;93 (2):199-208. doi:10.1189/jlb.0812429

28. Goyal A, Prasad R, Goel P, Pal A, Prasad S, Rani I. An integrated approach of the potential underlying molecular mechanistic paradigms of SARS-CoV-2-mediated coagulopathy. Indian $J$ Clin Biochem. 2021;15:1-17.

29. Tan Y, Zhang W, Zhu Z, et al. Integrating longitudinal clinical laboratory tests with targeted proteomic and transcriptomic analyses reveal the landscape of host responses in COVID-19. Cell Discov. 2021;7(1):1-19. doi:10.1038/s41421-021-00274-1

30. Byrne GJ, Ghellal A, Iddon J, et al. Serum soluble vascular cell adhesion molecule-1: role as a surrogate marker of angiogenesis. $J$ Natl Cancer Inst. 2000;92(16):1329-1336. doi:10.1093/jnci/ 92.16.1329

31. Xie H, Kang YJ. Role of copper in angiogenesis and its medicinal implications. Curr Med Chem. 2009;16(10):1304-1314. doi:10.2174/ 092986709787846622

32. Toh H, Cao M, Daniels E, Bateman A. Expression of the growth factor progranulin in endothelial cells influences growth and development of blood vessels: a novel mouse model. PLoS One. 2013;8 (5):e64989. doi:10.1371/journal.pone.0064989

33. Myhre PL, Heck SL, Skranes JB, et al. Cardiac pathology 6 months after hospitalization for COVID-19 and association with the acute disease severity: cardiac MRI 6 months after COVID-19. Am Heart J. 2021;13(21):00197.

34. Puntmann VO, Carerj ML, Wieters I, et al. Outcomes of cardiovascular magnetic resonance imaging in patients recently recovered from coronavirus disease 2019 (COVID-19). JAMA Cardiol. 2020;5 (11):1265-1273. doi:10.1001/jamacardio.2020.3557

35. Pour OB, Yahyavi Y, Karimi A, et al. Serum trace elements levels and clinical outcomes among Iranian COVID-19 patients. Int J Infect Dis. 2021;25(21):053. 
36. Rani I, Goyal A, Bhatnagar M, et al. Potential molecular mechanisms of zinc- and copper-mediated antiviral activity on COVID-19. Nutr Res. 2021;92:109-128. doi:10.1016/j.nutres.2021.05.008

37. Skalny AV, Rink L, Ajsuvakova OP, et al. Zinc and respiratory tract infections: perspectives for COVID-19 (Review). Int J Mol Med. 2020;46(1):17-26.

38. Bui TM, Wiesolek HL, Sumagin R. ICAM-1: a master regulator of cellular responses in inflammation, injury resolution, and tumorigenesis. J Leu Biol. 2020;108(3):787-799. doi:10.1002/ JLB.2MR0220-549R

39. Stubert J, Szewczyk M, Spitschak A, Knoll S, Richter DU, Pützer BM. Adenoviral mediated expression of anti-inflammatory progranulin by placental explants modulates endothelial cell activation by decrease of ICAM-1 expression. Placenta. 2020;90:109-117. doi:10.1016/j.placenta.2019.12.018
40. Manne BK, Denorme F, Middleton EA, et al. Platelet gene expression and function in patients with COVID-19. Blood. 2020;136 (11):1317-1329. doi:10.1182/blood.2020007214

41. Neri T, Nieri D, Celi A. P-selectin blockade in COVID-19-related ARDS. Am J Physiol Lung Cell Mol Physiol. 2020;318(6):L1237L1238. doi:10.1152/ajplung.00202.2020

\section{Publish your work in this journal}

The Journal of Inflammation Research is an international, peerreviewed open-access journal that welcomes laboratory and clinical findings on the molecular basis, cell biology and pharmacology of inflammation including original research, reviews, symposium reports, hypothesis formation and commentaries on: acute/chronic inflammation; mediators of inflammation; cellular processes; molecular mechanisms; pharmacology and novel anti-inflammatory drugs; clinical conditions involving inflammation. The manuscript management system is completely online and includes a very quick and fair peerreview system. Visit http://www.dovepress.com/testimonials.php to read real quotes from published authors. 